\title{
Characterisation of Channel Usage in ISM/SRD Bands
}

\author{
Hendrik Lieske $^{1}$, Frederik Beer ${ }^{1}$, Gerd Kilian ${ }^{2}$, Joerg Robert', Albert Heuberger ${ }^{1}$ \\ ${ }^{1}$ Friedrich-Alexander-Universität Erlangen-Nürnberg (FAU), \\ Information Technology (Communication Electronics), 91058 Erlangen, Germany, \\ Hendrik.Lieske@fau.de \\ ${ }^{2}$ Fraunhofer Institute for Integrated Circuits IIS, 91058 Erlangen, Germany
}

\begin{abstract}
:
Spectrum occupancy measurements for sub-GHz unlicensed frequency bands at two distinct inner city locations are presented in this paper. Short time measurements of spectrum utilization in three different bands are followed by a long-term observation of a selected sub-band over 7 days. The antenna in the latter measurement has an exposed position, which is typical for a fixed base station of a data aggregation network. For a selected frequency range, important signal characteristics (e.g. signal durations and inter-arrival times) are obtained and utilized to parameterise a proposed interference model. This analytic model accurately describes the collision probability of a packet transmission in the considered channel.
\end{abstract}

Key words: spectrum utilization measurement, unlicensed frequency band, interference modelling

\section{Introduction}

Applications using the unlicensed sub-GHz ISM (Industrial, Scientific and Medical) and SRD (Short Range Device) frequency bands for data transmission are getting more and more popular. Examples are smart car keys, small weather stations, or baby monitors. However, also operators offering commercial services are using these frequency bands as well. Largescale metering networks are one example, which have gained increasing interest over the recent years. Unfortunately, shared frequency resources are typically not used in a cooperative way, leading to inter- and intrasystem interference, resulting in increased packet collisions, and hence a reduced communication performance. Whilst this may still be tolerable in personal applications, commercial providers have to find means to quantify and mitigate the effects caused by interference.

Inter-system interference, i.e. interference caused by other communication systems in the same frequency band, may have a severe impact on communication performance. To assess this impact, statistical models describing the wireless channel utilization would be of immense help. Therefore, spectrum monitoring of unlicensed bands is required to determine the utilization of shared frequency resources, which in turn is the starting point for developing appropriate interference models.
Results from spectrum measurements are frequently appearing within literature. As an example, in [1] the spectrum usage in central London over an 8-day period is presented. [2] focusses on spectrum occupancy in indoor and outdoor environments in Aachen (Germany) for up to 7 days, and [3] even compares spectrum utilization in three different regions in Europe. All three documents have in common that broad frequency ranges (from $20 \mathrm{MHz}$ to up to $6 \mathrm{GHz}$ ) are explored. On the one hand, an entire overview of the whole spectrum is given; on the other hand wideband measurements imply higher sweep times, and thus decreased timeresolution, which makes them inapplicable for accurate physical layer interference models.

In order to obtain the characteristic of interfering signals, which are required for an interference model, we performed a high time resolution spectrum measurement campaign with focus on unlicensed frequency bands in the sub- $\mathrm{GHz}$ range, namely the $169 \mathrm{MHz}$ band (169.4$169.8125 \mathrm{MHz}$ ), the $433 \mathrm{MHz}$ band (433.05$434.79 \mathrm{MHz}$ ) and the $868 \mathrm{MHz}$ band (863$870 \mathrm{MHz}$ ). We then used the characteristics of the measures signals, such as duration and arrival rate, to parameterise an interference model.

The paper is organized as follows. In the next section, we present short-time measurements of all frequency bands from central Nuremberg (Germany). Having received an impression of current spectrum utilization in unlicensed 
bands, we continue with a 7 day long-term measurement of the upper $868 \mathrm{MHz}$ band in central Erlangen (Germany). Afterwards we examine the signal characteristic in an exemplary chosen sub-band and propose a corresponding interference model. The model is evaluated by comparison with simulation results based on the measured data.

\section{Short-time measurements in Nuremberg}

The first measurement campaign for getting a first glance of the spectral utilization in the 169, 433 and $868 \mathrm{MHz}$ bands took place in central Nuremberg at the roof of the Technische Hochschule Nürnberg (N49 ${ }^{\circ} 27^{\prime} 10.224 " \mathrm{E} 11^{\circ}$ $5^{\prime}$ 39.48"). The receiver was a professional IZT R3301 portable monitoring receiver with dedicated omni-directional and vertically polarized measurement antennas for each frequency band. In contrast to most measurement setups in related publications, we captured the complex baseband data, which allows deeper insight in the nature of the received signals. The power spectral density (PSD) was estimated from consecutive frames of length $\mathrm{N}$ samples using an $\mathrm{N}$ sample fast Fourier transform (FFT) weighted with a Blackman window. The short-time measurements from mid-September 2013 lasted two hours and covered the whole frequency range of each considered frequency band.

Fig 1 a) shows the maximum and mean power spectral density for the $868 \mathrm{MHz}$ Band. The receiver's centre frequency is denoted by $f_{c}$. The frequency axis is separated into several sub-bands reflecting the general frequency assignment for non-specific SRDs from the Federal Network Agency (Amtsblatt Vfg 40/2010). Using a $N=8192$ FFT and a sampling frequency of $\mathrm{f}_{\mathrm{S}}=10 \mathrm{MHz}$, the time resolution of each PSD is $819 \mu$ s and approx. 8.8 million PSDs have been obtained. With regard to the mean $P S D$, the concurrence of large sections with the expected noise floor at $-130 \mathrm{dBm}$ clearly indicates unutilized frequency resources. Significant activity is only visible in the sub-bands $863-865 \mathrm{MHz}, 868-868.6 \mathrm{MHz}$ and $868.7-869.2 \mathrm{MHz}$. The harsh and broad maximum power density curve indicates the occurrence of strong short-time interferers during observation, e.g. caused by impulsive noise resulting from switching effects. The maximum measured power level was $-72 \mathrm{dBm}$.

Another important metric is the relative occupancy of the observed band. We use an energy detector to decide whether a frequency bin, i.e. one FFT output frequency, is occupied or unused. If the power in a frequency bin is above a decision threshold $\mu$ the bin is stated occupied. Clearly, the choice of $\mu$ has a strong impact on the reported occupancy, and hence must be chosen carefully. We follow the recommendation in [4] and set $\mu$ to $10 \mathrm{~dB}$ above the noise floor. Fig. 2 b) depicts the relative occupancy for the $868 \mathrm{MHz}$ band. The overall band occupancy is only $1.43 \%$. However, very high activity can be observed in the sub-band $863-865 \mathrm{MHz}$ with a peak occupancy of up to $80 \%$. Obviously, reliable communication in this sub-band would be a challenging task.

Fig. 2 shows the results for the $433 \mathrm{MHz}$ band at the same location. The time resolution for each PSD was $1.3 \mathrm{~ms}$. The maximum received power level during the observation was about $-60 \mathrm{dBm}$. The mean PSD shows activity in most frequency bins, which indicate numerous participants in this band. However, significant occupancy above $40 \%$ has been reported only in three narrow channels. The overall occupancy was $2.66 \%$.

The $169 \mathrm{MHz}$ band, which is attractive for longrange telemetry applications due to higher wavelengths, showed no occupancy.

Although the overall occupancy in the $433 \mathrm{MHz}$ and $868 \mathrm{MHz}$ bands is below 3\%, high occupancies of up to $80 \%$ have been reported for selected channels. The short observation time for these results raises further questions: How does the receive power and occupancy evolve over longer measurement periods? Is there even a relation to day/night cycles or between week- and holidays? The answers are given with a seven day measurement presented in the next section.

\section{Long-term measurement in Erlangen}

The measurement setup of the long-term observation was as follows. A log-periodic and vertically polarized wideband antenna (KATHREIN 742 192V01) was situated at the top of a chimney (140 $\mathrm{m}$ above ground level) in central Erlangen $\left(\mathrm{N} 49^{\circ} 35^{\prime} 34.35^{\prime \prime}\right.$ E11 ${ }^{\circ} 0^{\prime}$ 7.985"). The professional fixed station antenna has a gain of $10.8 \mathrm{dBi}$ and points to southeastern direction. Its exposed site is typical for base stations of a large-scale data aggregation networks. The antenna was connected to a software defined radio platform (Universal Software Radio Peripheral N200 with SBX daughterboard), which continuously captured a $2 \mathrm{MHz}$ band between 868 and $870 \mathrm{MHz}$. An analogue prefilter $(863-870 \mathrm{MHz})$ was used to reduce the impact of nearby cellular (e.g. GSM, LTE) and broadcast service antennas. The received samples were streamed to a dedicated computer, which performed real-time estimation 

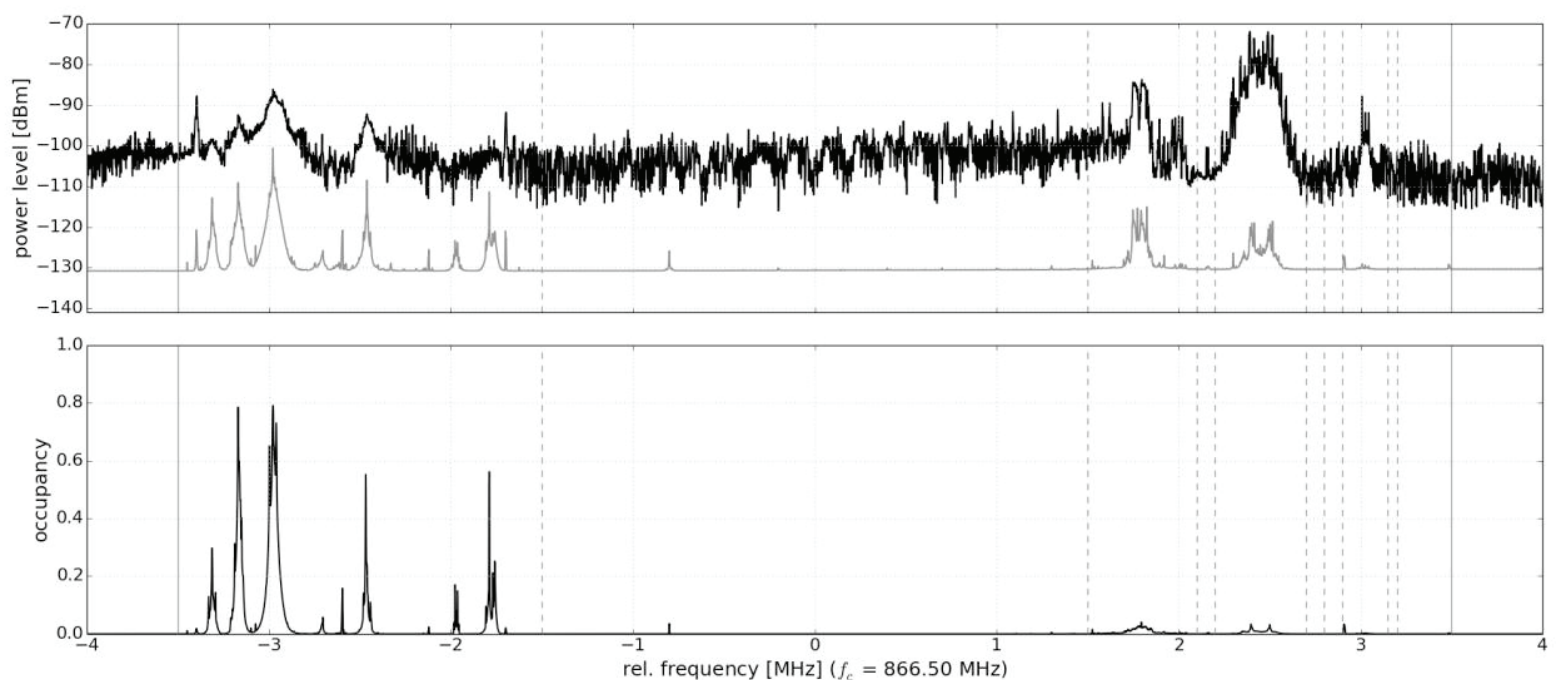

Fig. 1. Maximum and mean PSD (above) and relative occupancy (below) for the $868 \mathrm{MHz}$ band measured at central Nuremberg. Observation duration: $2 \mathrm{~h}$; Resolution bandwidth: $1.2 \mathrm{kHz}$
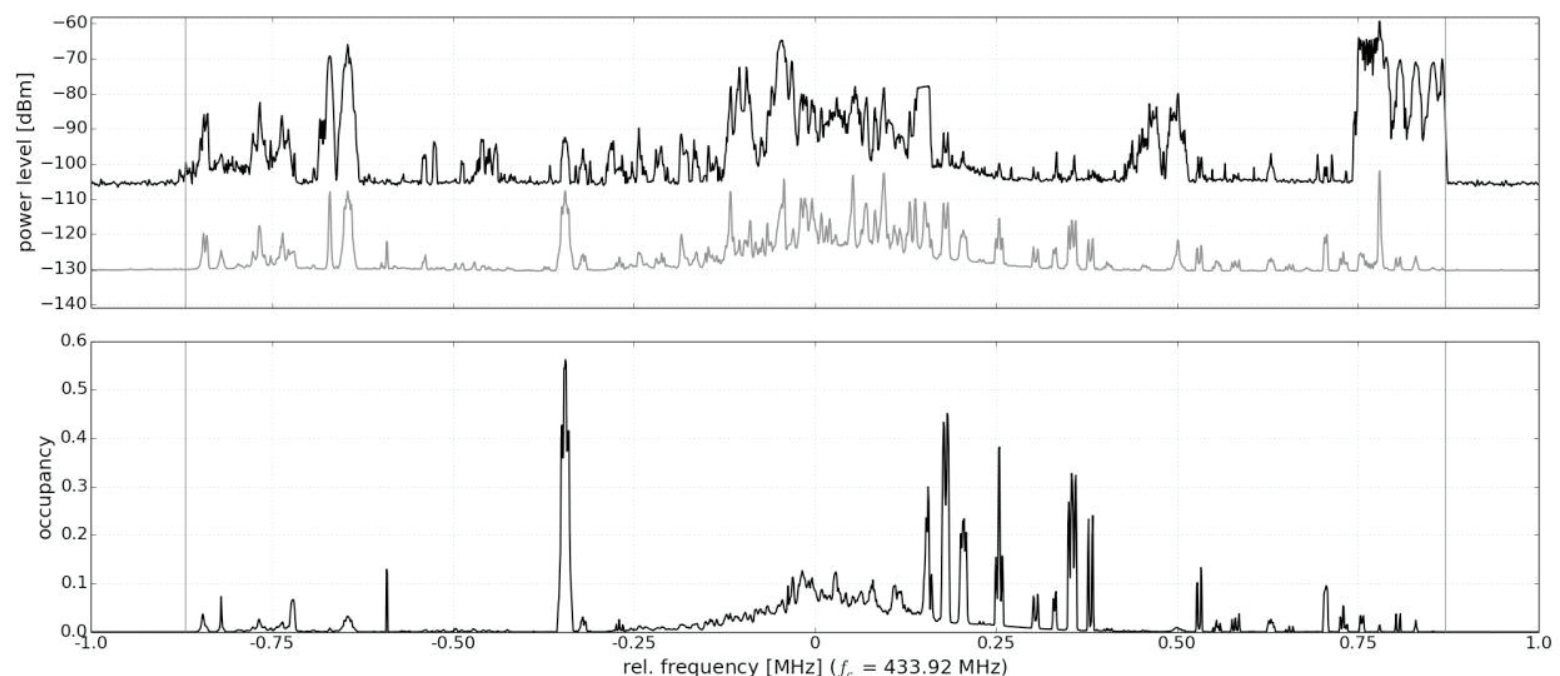

Fig. 2. Maximum and mean PSD (above) and relative occupancy (below) for the $433 \mathrm{MHz}$ band measured at central Nuremberg. Observation duration: $2 \mathrm{~h}$; Resolution bandwidth: $977 \mathrm{~Hz}$
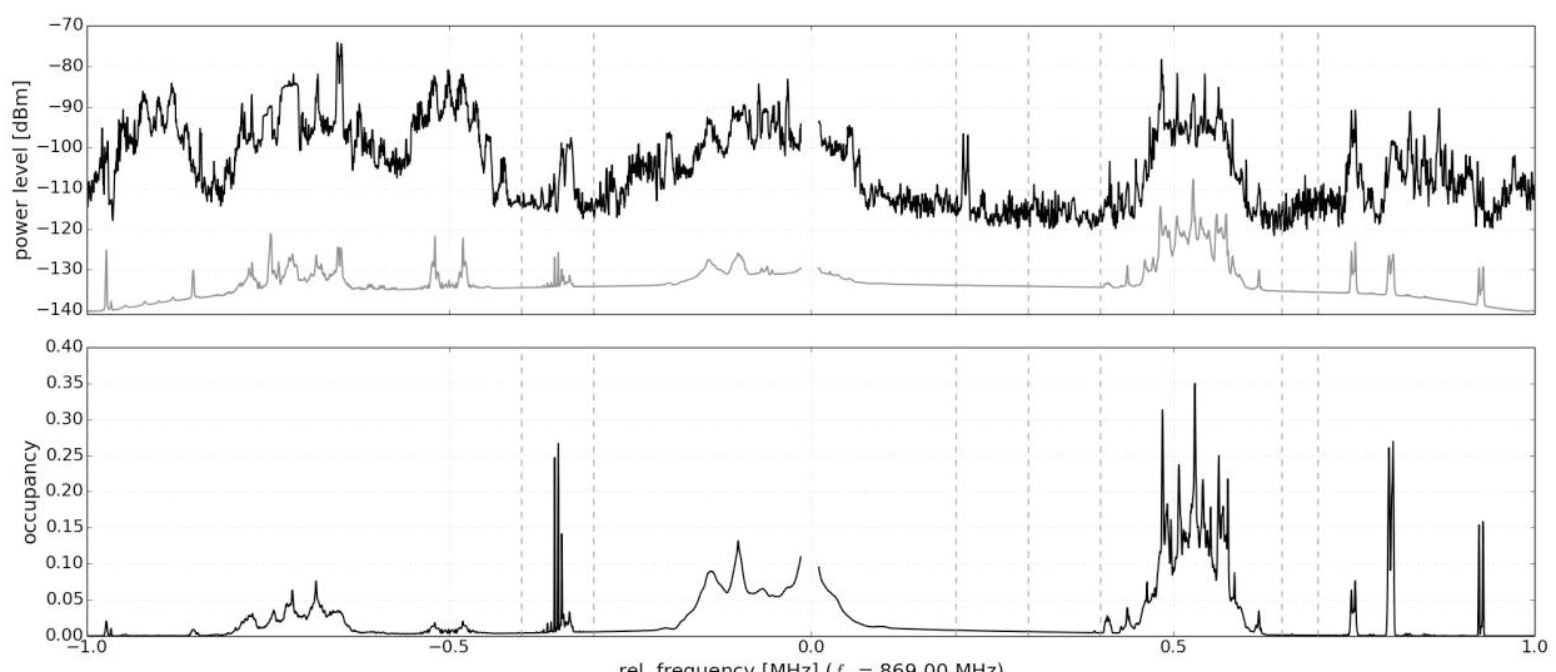

rel. frequency $[\mathrm{MHz}]\left(f_{c}=869.00 \mathrm{MHz}\right)$

Fig. 3. Maximum and mean PSD (above) and relative occupancy (below) for the upper $868 \mathrm{MHz}$ band measured at central Erlangen. DC component from receiver hardware was removed at $f=0 \mathrm{~Hz}$. Observation duration: $7 \mathrm{~d}$; Resolution bandwidth: $488 \mathrm{~Hz}$ 
of the instantaneous spectrum utilization. In general, a reliable data streaming is the bottleneck, which limited the sampling frequency and therefore the observed bandwidth to $2 \mathrm{MHz}$ in our case.

The measurement was conducted from 16.04.2014 to 23.04.2014 with a time resolution of $2.05 \mathrm{~ms}$ for each PSD. To keep the amount of acquired data manageable, the PSDs in a five minute time span were used to create an intermediate result - a so called report. Note that each report contains the maximum and mean received power for each PSD bin for the given report interval. The same applies for the spectrum occupancy. Altogether, 2024 reports have been created during the measurements.

Fig. 3 depicts the consolidated results over the seven day measurement. The maximum measured power level was $-74 \mathrm{dBm}$ at 868.3 MHz. The mean PSD has its distinct maximum in the sub-band $868.4-869.5 \mathrm{MHz}$. This coincides with the so-called high power band, where participants are allowed to transmit with an equivalent radiated power (ERP) of up to $500 \mathrm{~mW}$ when they apply spectrum access techniques like listen before talk or other interference mitigation techniques.

Fig 3 b) shows the overall spectrum occupancy. Similar to the results presented in the previous chapter, the total occupancy was about $2.29 \%$ (threshold $\mu=-127 \mathrm{dBm}$ ). Higher activity of above $15 \%$ can be observed in the high power band, in two narrow channels between 869.7$870 \mathrm{MHz}$, and surprisingly, in a dedicated band for alarm messages at 868.6 MHz. However, there was also a significant amount of spectrum that was not occupied by any transmission during the measurement campaign.

Next, we analyse the maximum power level and the occupancy for two distinct sub-bands over time. Therefore, each data point in the following plots represents a five minute report interval. Since a sub-band covers several frequency bins, the maximum value of all bins belonging to the sub-band was chosen, which represents a worst case scenario.

Fig. 4 shows the results over time for the high power band (869.4-869.65 MHz). It is notable that there are two time intervals with increased signal power: From the beginning to Thursday $6 \mathrm{pm}$ and from Friday evening to Saturday evening. The maximum channel occupancy is fairly high (approx. $35 \%$ ) and relatively constant. No relationship between the spectrum utilization and different days are visible.

The situation is different for the narrow subband between 869.7375 and $869.7625 \mathrm{MHz}$.
Fig. 5 shows a strong time dependency of the channel access. A high occupancy has been measured during usual work times from 7 am to $6 \mathrm{pm}$. Note that Friday and Monday were Easter holidays. On Tuesday, the occupancy even reaches $100 \%$ during mid-day hours.

\section{Signal characterization}

Having analysed the long-term behaviour over several days of two selected channels in the last section, we now focus on characterizing the received signals itself in an exemplarily chosen channel. Therefore, we consider a narrow frequency channel at the centre frequency 868.9 MHz with a bandwidth of $20 \mathrm{kHz}$, which has been measured on the chimney in Erlangen. This channel was selected since random access has been observed in a spectrogram plot. Complex baseband data for a time interval of one hour was analysed to estimate length, receive power and inter-arrival time of detected packet transmissions. Again, an energy detector was used to assess an occupied channel state. Since the packet detection in the given low signal-to-noise environment is a challenging task, two assumptions were made to distinguish between packet transmissions and noise:

- The minimum length of a packet is 500 us.

- The minimum mean power of a packet must exceed $-110 \mathrm{dBm}$ (10 dB above expected noise floor).

Consecutive baseband sample blocks, which fulfil both assumptions, were recognized as packet transmissions.

Overall, 17382 packets were identified. The estimated probability density functions for the three captured parameters are depicted in Fig. 6. Considering the packet length, the channel was obviously dominated by three different packet types of length $0.7 \mathrm{~ms}, 8 \mathrm{~ms}$ and $14 \mathrm{~ms}$, respectively.

\section{Interference channel model}

Finally, we take the results from the previous section to propose a model which describes the probability of interference in the considered channel. Such models are of great significance, e.g. during network planning. Using the derived model, the outage probability of a radio link can be estimated before installation, and system parameters such as packet length, duty cycle or medium access control algorithms can be adapted in advance to improve communication reliability.

In the following, we consider the identified packets in the last section as interference to a reference transmission with packet length $L_{\text {ref. }}$. 

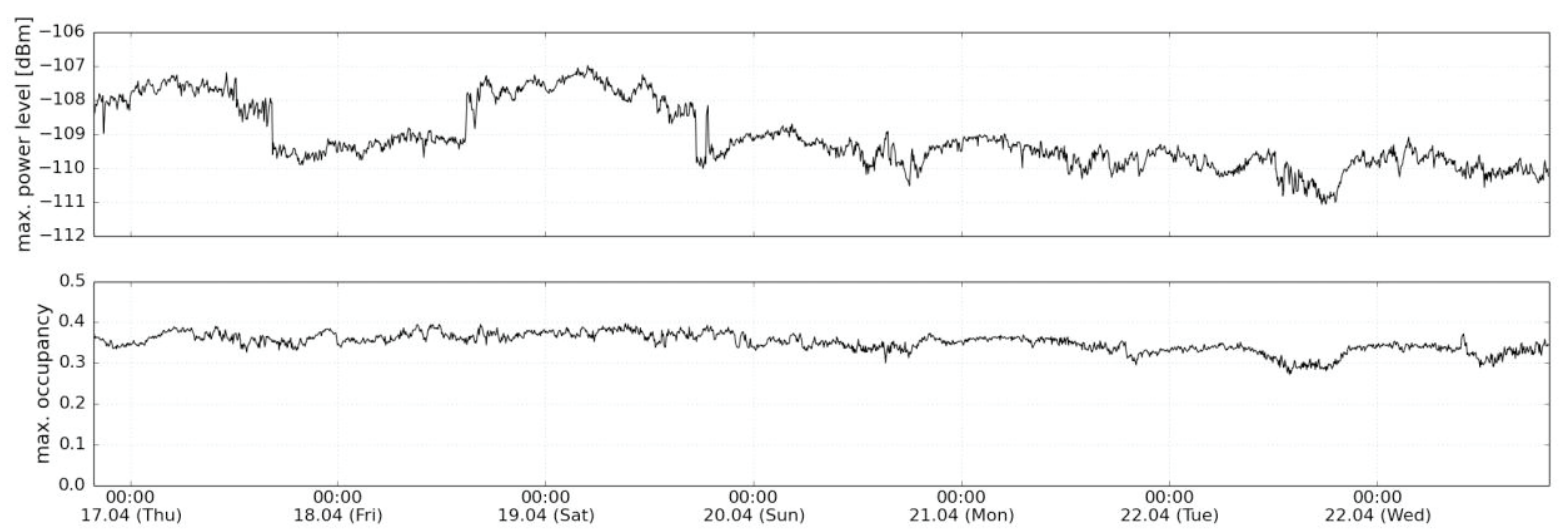

Fig. 4. Maximum receive power and maximum channel occupancy in channel $869.525 \pm 125 \mathrm{kHz}$ (Erlangen setup, high power band) over time. One sample point corresponds to report interval of $5 \mathrm{~min}$.
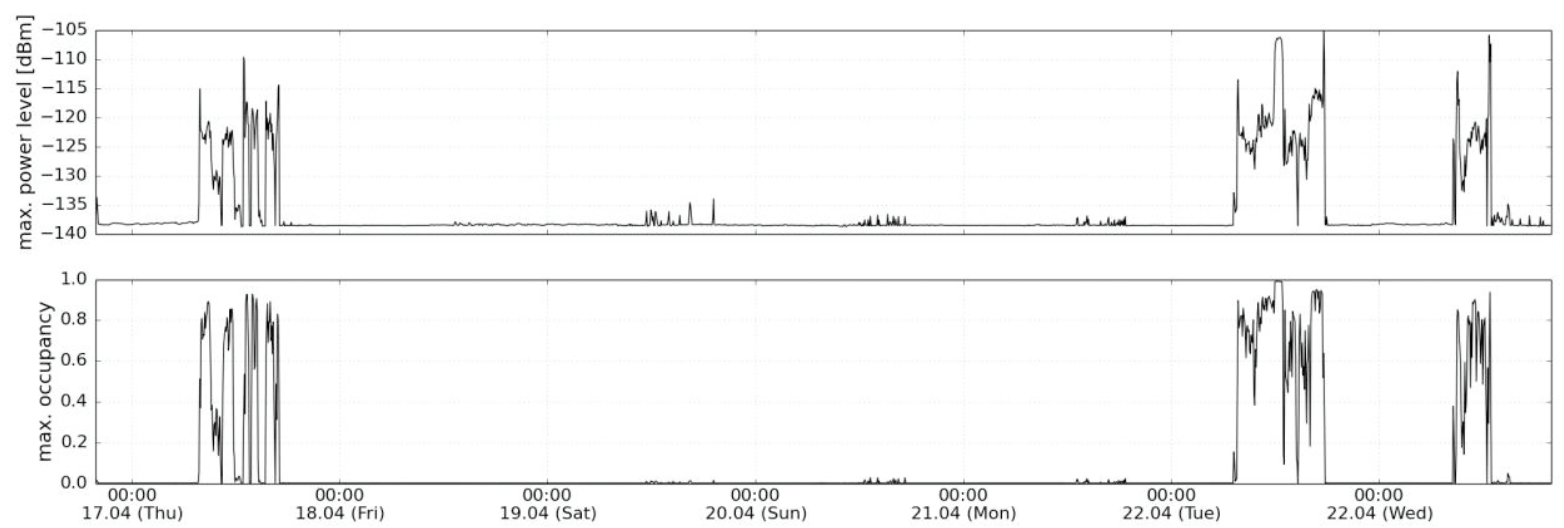

Fig. 5. Maximum receive power and maximum channel occupancy in channel $869.75 \mathrm{MHz} \pm 12.5 \mathrm{kHz}$ (Erlangen setup) over time. Friday and Monday are Easter holidays. One sample point corresponds to report interval of $5 \mathrm{~min}$.
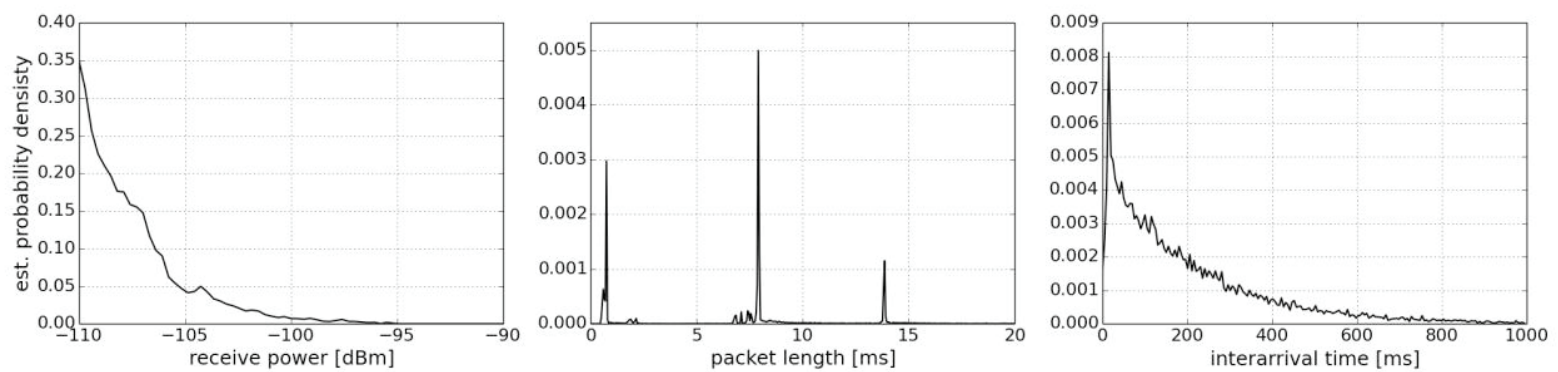

Fig. 6. Estimated pdfs of receive power, packet length and inter-arrival time of identified packets in frequency channel $868.9 \mathrm{MHz} \pm 10 \mathrm{kHz}$ (Erlangen setup). Observation length was one hour.

A common approach for modelling the arrival times of interferers is assuming a Poisson process [5], which implies an exponential distributed inter-arrival time between interferers with arrival rate $\lambda$. Since fig. $6 \mathrm{~b}$ ) indicates three different packet classes, the packets were grouped according to their length in three different sets: Set $A$ contains all packets within length $0.5 \mathrm{~ms} \leq \mathrm{L}<5 \mathrm{~ms}$, set $\mathrm{B}$ within $5 \mathrm{~ms} \leq \mathrm{L}<10 \mathrm{~ms}$, and set $\mathrm{C}$ within $10 \mathrm{~ms} \leq \mathrm{L}<20 \mathrm{~ms}$. In summary, these sets contain $99 \%$ of all detected packets. For each set the mean inter-arrival time $\lambda_{i}^{-1}$ and the mean packet length $L_{i}$ was computed ( $i \in\{A, B, C\}$ ). The results are given in table 1 .
Tab. 1: Mean inter-arrival time $\lambda_{i}^{-1}$ and mean packet length $L_{i}$ for the individual packet sets $A, B$ and $C$.

\begin{tabular}{|c|c|c|}
\hline Set $\mathrm{i}$ & $\lambda_{i}^{-1}$ & $\mathrm{~L}_{i}$ \\
\hline $\mathrm{A}$ & $703.8 \mathrm{~ms}$ & $0.9 \mathrm{~ms}$ \\
\hline$B$ & $404.0 \mathrm{~ms}$ & $7.9 \mathrm{~ms}$ \\
\hline $\mathrm{C}$ & $1072.0 \mathrm{~ms}$ & $14.5 \mathrm{~ms}$ \\
\hline
\end{tabular}

Next, we consider the special case of one interferer with packet length L. Assuming a Poisson distribution for the arrival process of interfering transmissions, the probability $\mathrm{P}_{\text {suc }}$ of 
the event that the reference transmission with length $L_{\text {ref }}$ will not interfere is given by [6]:

$$
\mathrm{P}_{\text {suc }}=\mathrm{e}^{-\left(\mathrm{L}_{\mathrm{ref}}+\mathrm{L}\right) \cdot \lambda} \text {. }
$$

Let each set i describe a self-standing interferer with length $L_{i}$ and arrival rate $\lambda_{i}$. Assuming the occurrence of these interferers $A, B$ and $C$ as statistically independent, the probability $P_{\text {col }}$ of the event that the reference transmission collides with at least one interferer can be expressed as:

$$
\mathrm{P}_{\text {col }}=1-\prod_{\mathrm{i}} \mathrm{e}^{-\left(\mathrm{L}_{\mathrm{ref}}+\mathrm{L}_{\mathrm{i}}\right) \cdot \lambda_{\mathrm{i}}} \mathrm{i} \in\{\mathrm{A}, \mathrm{B}, \mathrm{C}\} \text {. }
$$

The red curve in Fig. 7 shows the theoretic collision probability over varying packet lengths $L_{\text {ref }}$ for the extracted interferer parameters in table 1.

We now analyse how this theoretic model reflects the actually measured channel conditions. For this purpose, we consider a discrete time signal $x[n]$ that indicates whether the channel is currently occupied or not. This signal is the binary counterpart to the complex baseband signal that has been used in the previous section to obtain the signal characteristics given in Table 1 . The sampling rates $f_{s}$ of both signals are identical. $x[n]$ is 1 if a packet transmission at time instance $n$ has been detected and 0 otherwise. For simulating the collision probability with a reference transmission of length $L_{\text {ref, a vector of length }}$ $\left\|L_{\text {ref }} \cdot f_{s}\right\|$ - where $\|\cdot\|$ denotes rounding to nearest integer - at any time instant $n$ is considered. If this vector indicates any occupied channel state, a collision is assumed. The number of collisions divided by the total number of considered time instants leads to the collision probability. The simulation results for the measured channel state signal $x[n]$ are depicted by the red markers in Fig. 7 .

Both curves almost coincide, which proofs that the assumptions for the theoretic model are valid and (2) describes the collision probability for the given channel with high accuracy. It is worth to note that the channel occupancy is only $3.37 \%$. However, the collision probability of a transmission with length $40 \mathrm{~ms}$ is already over $20 \%$.

A key factor in setting up the model is the distinction between noise and burst transmissions inside the captured complex baseband signal. Depending on the communication system under consideration, the assumptions made in the previous section to identify bursts must be carefully reviewed. In addition, the model presumes random channel access, which may not be the case in channels with more regular data transmission. Consider e.g. a typical Wi-Fi channel: A successful data transmission packet will be followed by a short inter-frame spacing and an acknowledge message. The fixed relationship between consecutive transmissions would require a more complex interference model.

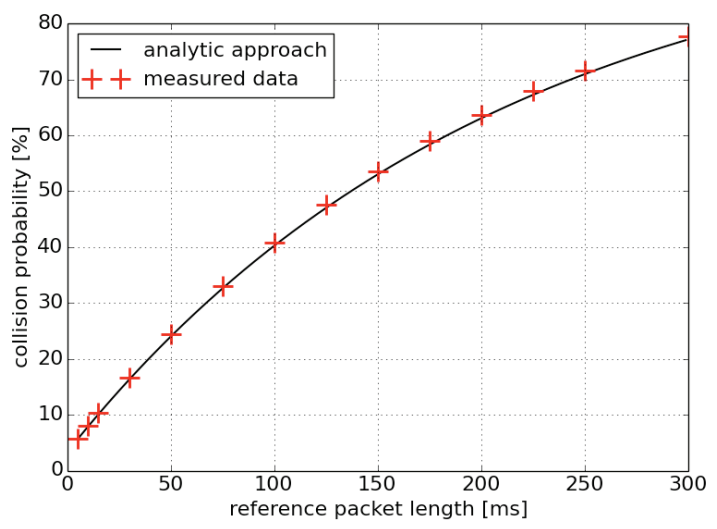

Fig. 7. Collision probability of a reference transmission with packet length $L_{\text {ref }}$ in the considered channel.

\section{Conclusions}

This paper offered insight in the current spectrum utilization of sub-GHz unlicensed frequency bands at inner city areas. Two hour measurements for three different bands were presented, which showed considerable occupancy in selected sub-bands for the $868 \mathrm{MHz}$ and $433 \mathrm{MHz}$ band. However, the total band occupancy is still low at about $3 \%$. No activity has been reported in the $169 \mathrm{MHz}$ band. The long-term behaviour has been analysed with a seven day measurement of the upper $868 \mathrm{MHz}$ band. Finally, we analysed the signal characteristics and provided measured distributions of receive power, signal length and interarrival times. These results were used to parameterize a proposed interference model, which accurately describes the collision probability in the considered frequency channel.

The good fit of the analytic interference model with the measured data raises the question, how this model will perform in more heterogeneous channels, i.e. with additional participants that access the channel periodically. A thorough analysis of such channels and the derivation of suitable interference models is subject of future work.

\section{Acknowledgment}

The work has been partly supported by EFRE funding from the Bavarian Ministry of Economic Affairs (Bayerisches Staatsministerium für Wirtschaft, Infrastruktur, Verkehr und Technologie) as a part of the "ESI Application Center" project. We also would like to thank our 
students Sebastian Hagl and Sebastian Luber for their contribution.

\section{References}

[1] A. Palaios, J. Riihijarvi, O. Holland, P. Mahonen, A week in London: Spectrum usage in metropolitan London, Personal Indoor and Mobile Radio Communications (PIMRC), IEEE 24th International Symposium on (2013); doi: 10.1109/PIMRC.2013.6666571

[2] M. Wellens, J. Wu, P. Mahonen, Evaluation of spectrum occupancy in indoor and outdoor scenario in the context of cognitive radio, Cognitive Radio Oriented Wireless Networks and Communications, CrownCom 2007. 2nd International Conference on (2007); doi: 10.1109/CROWNCOM.2007.4549835

[3] V. Valenta, R. Maršálek, G. Baudoin, M. Villegas, M. Suarez, F. Robert, Survey on spectrum utilization in Europe: Measurements, analyses and observations, Cognitive Radio Oriented Wireless Networks Communications (CROWNCOM), Proceedings of the Fifth International Conference on (2010); doi: 10.4108/ICST.CROWNCOM2010.9220

[4] Radiocommunications Bureau, Handbook spectrum monitoring, Technical Report, International Telecommunication Union (ITU), 2011

[5] V. Frost, B. Melamed, Traffic modeling for telecommunications networks, Communications Magazine, IEEE, 1994; doi: 10.1109/35.267444

[6] G.Kilian, M. Breiling, H. Petkov, J. Robert, F.Beer, H.Lieske, A. Heuberger, Increasing Transmission Reliability for Telemetry Systems using Telegram Splitting, submitted for publication 